\title{
Clinical experience with the novel histone deacetylase inhibitor vorinostat (suberoylanilide hydroxamic acid) in patients with relapsed lymphoma
}

\author{
OA O'Connor*,I \\ 'Laboratory of Experimental Therapeutics, Department of Medicine, Division of Hematologic Oncology, Lymphoma Service, Memorial Sloan Kettering \\ Cancer Center, New York, NY, USA
}

\begin{abstract}
Preclinical studies indicate that vorinostat (suberoylanilide hydroxamic acid or SAHA) inhibits histone deacetylase (HDAC) activity, increases acetylated histones $\mathrm{H} 2 \mathrm{a}, \mathrm{H} 2 \mathrm{~b}, \mathrm{H} 3$, and $\mathrm{H} 4$, and thereby induces differentiation and apoptosis in a variety of tumour cell lines, including murine erythroleukaemia, human bladder transitional cell carcinoma, and human breast adenocarcinoma. On the basis of these favourable preclinical findings, vorinostat has been selected as a candidate for clinical development with the potential to treat patients with selected malignances, including Hodgkin's disease and non-Hodgkin's lymphomas. Phase I clinical trials in patients with haematological malignances and solid tumours showed that both intravenous (i.v.) and oral formulations of vorinostat are well tolerated, can inhibit HDAC activity in peripheral blood mononuclear cells and tumour tissue biopsies, and produce objective tumour regression and symptomatic improvement with little clinical toxicity. The dose-limiting toxicities (DLT) of i.v. vorinostat were primarily haematologic and were rapidly reversible within 4-5 days of therapy cessation. In contrast, the DLT for oral vorinostat were primarily non-haematologic (including dehydration, anorexia, diarrhoea, fatigue) and were also rapidly reversible, usually within 3 days. Further research is warranted to optimise the dosing schedule for vorinostat, particularly with respect to dose, timing of administration, and duration of therapy, and to fully delineate the mechanism(s) of antitumour effect of vorinostat in various types of malignances. Several phase II studies are currently ongoing in patients with haematological malignances and solid tumours.

British Journal of Cancer (2006) 95, S7-SI2. doi:I0.1038/sj.bjc.6603464 www.bjcancer.com
\end{abstract}

(C) 2006 Cancer Research UK

Keywords: histone deacetylase; non-Hodgkin's lymphoma; vorinostat; SAHA; Hodgkin's disease; BCL-6

The potential therapeutic benefit created by facilitated gene transcription has led to the development of histone deacetylase (HDAC) inhibitors, which have the capacity to induce cytodifferentiation, and cause cell-cycle arrest and apoptosis of transformed cells (Marks et al, 2004). One such HDAC inhibitor, vorinostat (suberoylanilide hydroxamic acid or SAHA), has been shown to induce differentiation of murine erythroleukaemia (Richon et al, 1996), human bladder transitional cell carcinoma (T24) (Richon et al, 2000), and human breast adenocarcinoma (MCF-7) (Huang and Pardee, 2000; Munster et al, 2001). Vorinostat inhibits HDAC through a direct interaction with the enzymes catalytic site, resulting in accumulation of acetylated histones $\mathrm{H} 2 \mathrm{a}, \mathrm{H} 2 \mathrm{~b}, \mathrm{H} 3$, and H4. Based on the biological rationale and early preclinical data summarised above, vorinostat was selected as a candidate for clinical development. This article will summarise the toxicity, pharmacokinetic and pharmacodynamic profiles, and efficacy of intravenous (i.v.) and oral formulations of vorinostat, and review

\footnotetext{
*Correspondence: Dr OA O'Connor, Laboratory of Experimental Therapeutics, Department of Medicine, Division of Hematologic Oncology, Lymphoma Service, Memorial Sloan Kettering Cancer Center, 1275 York Avenue (Box 329), New York I002I, USA.

E-mail: oconnoro@mskcc.org
}

the findings from initial phase I studies that revealed interesting differences in toxicity profile between the two formulations. Additional reviews regarding the biological aspects of HDACs can be found elsewhere (Marks et al, 1999; Marks et al, 2000; Marks et al, 2001; Dokmanovic and Marks, 2005; Marks and Dokmanovic, 2005).

\section{BCL-6 AS A THERAPEUTIC TARGET-RATIONALE FOR USE OF VORINOSTAT TO TREAT LYMPHOMA}

The proto-oncogene BCL-6, located at chromosome 3q27, encodes a $\mathrm{POZ} /$ zinc finger sequence-specific transcriptional repressor and represents one of three genes commonly implicated in nonHodgkin's lymphoma (NHL)(the other two genes are BCL-2 and c-myc) (Ye et al, 1993a, b; Lo et al, 1994; Kramer et al, 1998; Pasqualucci et al, 2003). BCL-6 is constitutively expressed in a large proportion of B-cell lymphomas where it appears to suppress genes involved in the control of lymphocyte activation, differentiation, and apoptosis (Pasqualucci et al, 2003). Recent studies suggest that the transcriptional co-activator p300 acetylates BCL-6 and, in doing so, disrupts the ability of BCL-6 to recruit HDACs, thereby compromising its ability to repress transcription and induce cell transformation (Bereshchenko et al, 2002). This has important therapeutic ramifications in that pharmacological 
Targeting BCL-6 in lymphoma

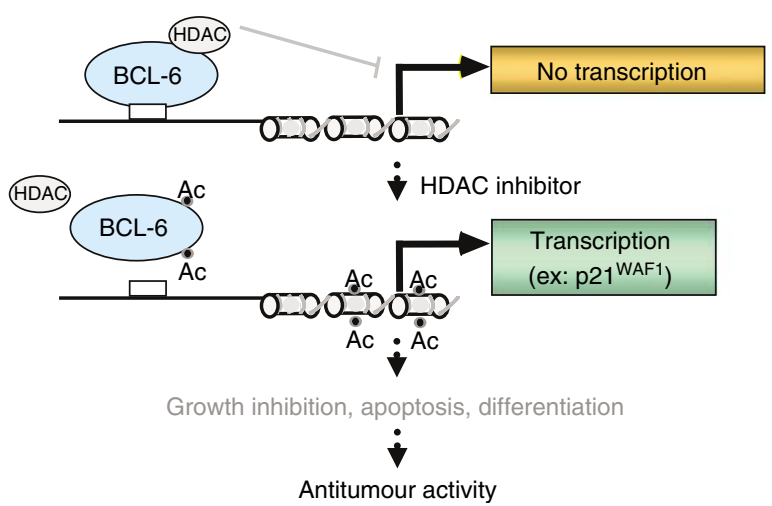

Figure I BCL-6 is constitutively expressed in a large proportion of B-cell lymphomas and is involved in the suppression of genes involved in the control of lymphocyte activation, differentiation, and apoptosis. In the acetylated state, BCL-6 is inactive and loses its ability to repress transcription and to induce cell transformation. Pharmacological inhibition of HDAC with agents such as vorinostat may result in the accumulation of acetylated $\mathrm{BCL}-6$, expression of the cell-cycle regulator $\mathrm{p} 2 \mathrm{I}^{\mathrm{WAFI}}$ and ultimately growth inhibition, apoptosis, and differentiation of B-cell lymphoma cells.

inhibition of HDACs might be expected to result in the accumulation of the inactive acetylated BCL- 6 which can then induce cell-cycle arrest and death of B-cell lymphoma cells (Figure 1). This hypothesis is supported by the observation that the HDAC inhibitor trichostatin A (TSA) results in apoptosis in several cell lines of B-NHL(Pasqualucci et al, 2003). Collectively, these studies suggest that HDAC inhibitors such as vorinostat may represent a novel therapeutic approach to treat patients with certain types of lymphomas.

\section{INTRAVENOUS AND ORAL FORMULATIONS OF VORINOSTAT}

Dose-escalating studies with daily 2-h i.v. infusions of vorinostat administered 5 days per week for up to 3 consecutive weeks demonstrated that an i.v. formulation could be administered safely to patients with haematological and solid tumours (Kelly et al, 2003). Peak vorinostat concentrations occurred approximately $60 \mathrm{~min}$ after beginning the infusion (range 25-120 min). The terminal half-life of i.v. vorinostat ranged from 21 to $58 \mathrm{~min}$, and the area under the plasma concentration curve was proportional to the dose administered. At all i.v. doses of vorinostat studied (75, $150,300,600$, and $900 \mathrm{mg} \mathrm{m}^{-2} \mathrm{day}^{-1}$ ), plasma concentrations exceeded $2.5 \mu \mathrm{M}$ (Figure 2), a concentration that inhibits cell proliferation in vitro and results in accumulation of acetylated histones. In addition, i.v. vorinostat was also shown to inhibit HDAC activity in normal cells (peripheral blood mononuclear cells) as well as in tumour tissue biopsies. As illustrated in three patients, accumulation of acetylated histones in peripheral blood mononuclear cells occurred at the end of a 2-h vorinostat infusion and was still evident $2 \mathrm{~h}$ after the infusion ended (Figure 3 ).

To simplify the administration of vorinostat, an oral formulation was developed, which also shows a favourable pharmacokinetic profile while retaining its antitumour activity. The bioavailability of oral vorinostat is relatively high $(43 \%)$ and appears to be uninfluenced by the consumption of food (Kelly et al, 2005). Oral vorinostat demonstrated linear pharmacokinetics from 200 to $600 \mathrm{mg}$, although peak concentrations were lower than that seen with the i.v. formulation (Kelly et al, 2005). The apparent half-life of oral vorinostat ranged from 91 to $127 \mathrm{~min}$, values that

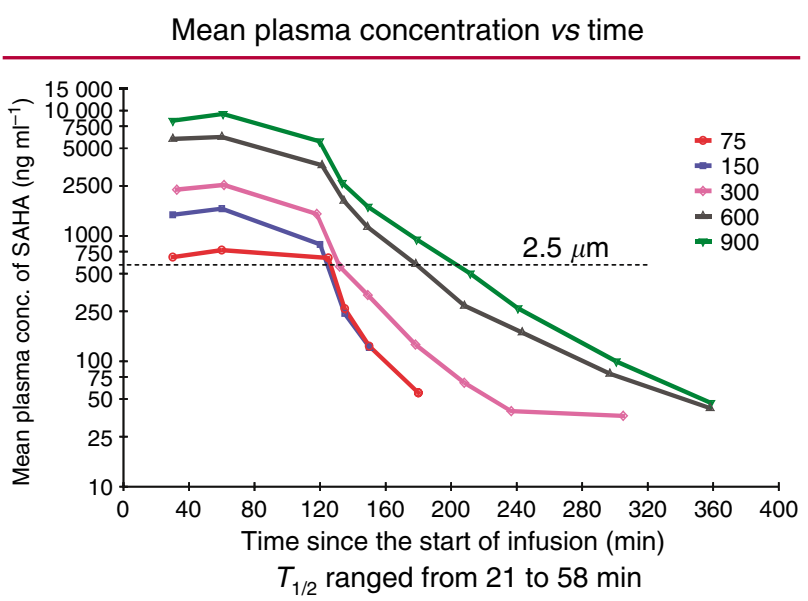

Figure 2 Mean plasma vorinostat concentrations over time following escalating i.v. vorinostat doses in patients with advanced cancer. Reproduced with permission from Kelly et al (2003).

Histone acetylation on Day 1 and Day 15 of treatment (300 $\mathrm{mg} \mathrm{m}^{-2}$ I.V. $\times 5$ days $\times 3$ weeks)

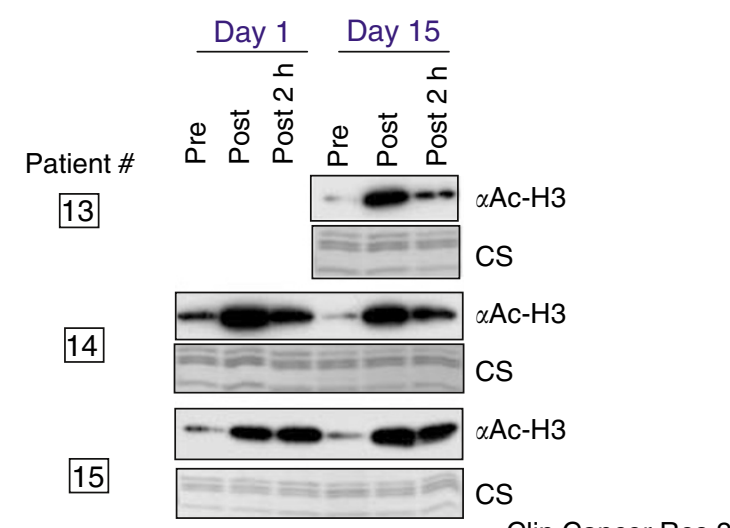

Clin Cancer Res 2003

Figure 3 Effect of i.v. vorinostat on histone acetylation in mononuclear cells (by Western blot analysis using a rabbit purified polyclonal antiacetylated histone $\mathrm{H} 3$ antibody) obtained from three cancer patients. As a loading control for histone proteins, parallel gels were run and stained with Coomassie (CS). Peripheral whole blood was collected from each patient before (pre) and at the end of a 2 -h vorinostat infusion (post), as well as $2 h$ after completion of infusion (post $2 \mathrm{~h}$ )

were 2-3-fold higher than those determined after i.v. administration. Plasma concentrations of vorinostat were detectable at least $10 \mathrm{~h}$ post-ingestion, whereas vorinostat was undetectable in plasma $4-6$ h after i.v. dosing. As observed following i.v. administration, oral vorinostat consistently effected accumulation of acetylated histones in peripheral blood mononuclear cells at $2 \mathrm{~h}$ post-dosing, an effect that persisted for up to $10 \mathrm{~h}$ after a single dose of $400 \mathrm{mg}$ or higher (Kelly et al, 2005). Histone acetylation was still apparent in patients receiving oral vorinostat for 6 months or longer.

\section{COMPARISON OF ORAL AND I.V. VORINOSTAT IN PHASE I STUDIES}

The antitumour effects of oral and i.v. formulations of vorinostat have been assessed in two Phase I clinical trials (Kelly et al, 2003, 2005). In both of these trials, escalation of vorinostat was carried out in parallel and independently in patients with solid 
Table I Summary of patient characteristics in i.v. and oral vorinostat groups

\begin{tabular}{lcc}
\hline & i.v. vorinostat & Oral vorinostat \\
\hline Total number of patients & I 4 Registered & 25 Registered \\
Male & I2 Treated & 23 Treated \\
Female & $7(58 \%)$ & $16(69 \%)$ \\
Median age & $5(42 \%)$ & $7(30 \%)$ \\
Diseases & $39(19-77)$ & $57(20-99)$ \\
Hodgkin's disease & $5(42 \%)$ & $7(30 \%)$ \\
DLBCL & $5(42 \%)$ & $7(30 \%)$ \\
Small lymphocytic & $1(8 \%)$ & $1(4 \%)$ \\
Mantle cell NHL & - & $2(9 \%)$ \\
CTCLPTCL & $1(8 \%)$ & $2(9 \%)$ \\
Multiple myeloma & - & $2(9 \%)$ \\
APL/MDS & $7(4-15)$ & $1(4 \%) / I(4 \%)$ \\
Prior chemotherapy or & & $5(1-11)$ \\
biological therapies per patient & & \\
(median) & $6(50 \%)$ & $8(35 \%)$ \\
Patients who received prior & & \\
stem cell transplant & lymphoma and one & lymphoma and one \\
Received rituximab & Hodgkin's disease) & Hodgkin's disease) \\
\hline
\end{tabular}

$\mathrm{APL}=$ acute promyelocytic leukaemia; $\mathrm{CTCL}=$ cutaneous $\mathrm{T}$-cell lymphoma; $\mathrm{DLBCL}=$ diffuse large B-cell lymphoma; i.v. = intravenous; $M D S=$ myelodysplastic syndrome; $\mathrm{NHL}=$ non-Hodgkin's lymphoma; $\mathrm{PTCL}=$ peripheral $\mathrm{T}$-cell lymphoma; $\mathrm{SAHA}=$ suberoylanilide hydroxamic acid.

tumours and haematological malignances. The present review primarily focuses on data obtained in patients with advanced haematological malignances who had failed or relapsed following standard therapy (O'Connor et al, 2006). The patient population included those with refractory or relapsed leukaemia, multiple myeloma, indolent or aggressive NHL, mantle cell lymphoma, and Hodgkin's disease (HD). Eligible patients were required to have a Karnofsky performance status of at least 70, adequate renal and hepatic function, an absolute neutrophil count of

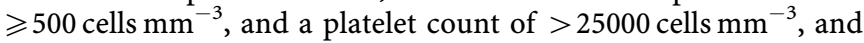
no serious comorbidities.

A total of 39 patients with haematological malignancy were registered, of which 35 were treated with either i.v. vorinostat or oral vorinostat (Table 1). Compared with the i.v. vorinostat group, patients receiving oral vorinostat showed a greater variation in the types of underlying haematological malignancy. The majority of patients in both groups were heavily pretreated, with some patients having received up to 15 different chemotherapy or biological therapies before study enrolment. In addition, between 35 and $50 \%$ of patients had received prior stem cell transplantation and approximately half of all patients had previously received therapy with rituximab. The duration of therapy was longer in patients receiving oral vorinostat compared with patients receiving i.v. vorinostat (average 14.1 vs 10.5 weeks, respectively). However, the largest difference between the study groups was age, with patients receiving i.v. vorinostat being considerably younger than patients receiving oral vorinostat (median age 39 vs 57 years, respectively).

\section{SAFETY AND TOLERABILITY}

Overall, both vorinostat formulations were well tolerated (Kelly et al, 2003, 2005; O'Connor et al, 2006). Major adverse events with oral vorinostat included fatigue, diarrhoea, anorexia, and dehydration, whereas myelosuppression and thrombocytopenia were more prominent with i.v. vorinostat (Table 2). Typically, the haematological toxicities, especially the thrombocytopenia, resolved shortly after vorinostat therapy was stopped, suggesting
Table 2 Common overall non-haematologic and haematological toxicities (grades 3 and 4)

\begin{tabular}{|c|c|c|c|c|}
\hline & \multicolumn{2}{|c|}{$\begin{array}{l}\text { i.v. vorinostat } \\
(n=12)\end{array}$} & \multicolumn{2}{|c|}{$\begin{array}{l}\text { Oral vorinostat } \\
\qquad(n=23)\end{array}$} \\
\hline & Grade 3 & Grade 4 & Grade 3 & Grade 4 \\
\hline & \multicolumn{4}{|c|}{ Non-haematological toxicities } \\
\hline Anorexia & 0 & 0 & 3 & 0 \\
\hline Constipation & I & 0 & 0 & 0 \\
\hline Diarrhoea & I & 0 & 6 & 0 \\
\hline Dehydration & 0 & 0 & | | & 0 \\
\hline Dyspnea & 2 & 0 & 0 & 0 \\
\hline Fatigue & 2 & 0 & 5 & 0 \\
\hline Infection w/o neutropenia & l & 0 & 5 & 0 \\
\hline Hyperglycemia & 0 & 0 & 3 & 0 \\
\hline Hypocalcemia & 0 & 0 & 1 & 0 \\
\hline Hypokalemia & 0 & 0 & 2 & 0 \\
\hline Hypophosphatemia & I & 0 & 4 & 0 \\
\hline Neurological, Guillan Barre & 0 & 0 & 1 & 0 \\
\hline Pleuritic chest pain & 1 & 0 & 0 & 0 \\
\hline \multirow[t]{2}{*}{ Thrombosis } & 1 & 0 & 1 & 0 \\
\hline & \multicolumn{4}{|c|}{ Haematological toxicities } \\
\hline Anaemia & 5 & 0 & 4 & 3 \\
\hline Leukopenia & I & I & 3 & 1 \\
\hline Neutropenia & 0 & 1 & 3 & 1 \\
\hline Thrombocytopenia & I & 0 & 7 & 1 \\
\hline
\end{tabular}

i.v. $=$ intravenous, SAHA = suberoylanilide hydroxamic acid

that toxicity did not affect megakaryocytes directly, but may have add an affect on the terminal budding stage of platelet formation. This was confirmed on evaluation of bone marrow biopsy specimens obtained at the platelet nadir, which demonstrated ample numbers of megakaryoctyes, most of which had actually lost their characteristic appearance, and failed to demonstrate evidence of terminal budding. There were no incidents of neutropenic fever or neutropenic sepsis.

The number of patients with dose-limiting toxicities (DLTs), defined as either grade 3 or 4 non-haematologic toxicities or treatment delay for toxicity for longer than 1 week, for each dose level in the i.v. or oral vorinostat groups are summarised in Table 3. Dehydration, diarrhoea, and fatigue were the predominant DLTs in patients receiving oral vorinostat. The $400 \mathrm{mg}$ daily oral dose was the best tolerated, with only $18 \%$ of patients experiencing DLT. In patients receiving i.v. vorinostat, neutropenia or thrombocytopenia as well as dehydration and diarrhoea were the most frequent DLTs.

\section{ANTITUMOUR ACTIVITY}

Intravenous vorinostat resulted in antitumour activity in three patients with HD who had previously failed autologous transplants (Table 4) (Kelly et al, 2003, 2005; O'Connor et al, 2006). One HD patient, who was maintained on vorinostat at a dose of $300 \mathrm{mg} \mathrm{m}^{-2}$ for 9 months, showed stabilisation of her disease on a computed tomography (CT) scan and normalisation of her positron emission tomography (PET) scan. This patient subsequently converted to oral vorinostat for ease of therapy. The second HD patient receiving i.v. vorinostat at a dose of $600 \mathrm{mg} \mathrm{m}^{-2}$ showed a $15 \%$ shrinkage of disease, which was associated with a marked improvement in her pulmonary and overall performance status (Figure 4). Her disease subsequently progressed during a period of therapy cessation owing to secondary systemic infection. The third HD patient demonstrated an approximately $42 \%$ regression of their disease (durable for 2 months) with symptomatic improvement. 
Table 3 Dose-limiting toxicities (grade 3 or 4 non-haematologic toxicities or treatment delay for toxicity for longer than I week) associated with i.v. or oral vorinostat therapy

i.v. vorinostat

\begin{tabular}{|c|c|c|c|}
\hline Dose level & Dose-limiting toxicities & Dose level & Dose-limiting toxicities \\
\hline $\begin{array}{l}300 \mathrm{mg} \mathrm{m}^{-2} \text { day }^{-1} \times 5 \text { day } \times 3 \text { weeks } \\
(n=7)\end{array}$ & None & $400 \mathrm{mg}$ once daily $(n=11)$ & Dehydration/diarrhoea/fatigue $(n=2)$ \\
\hline $\begin{array}{l}600 \mathrm{mg} \mathrm{m}^{-2} \text { day }^{-1} \times 5 \text { day } \times 3 \text { weeks } \\
(n=5)\end{array}$ & $\begin{array}{l}\text { Treatment delay for neutropenia or } \\
\text { thrombocytopenia }(n=2)\end{array}$ & 400 mg twice daily $(n=3)$ & Dehydration/fatigue/anorexia $(n=3)$ \\
\hline $600 \mathrm{mg}$ once daily $(n=3)$ & Dehydration/diarrhoea $(n=2)$ & $\begin{array}{l}600 \mathrm{mg} \text { once daily }(n=3) \\
200 \mathrm{mg} \text { twice daily }(n=6)\end{array}$ & $\begin{array}{l}\text { Dehydration/fatigue/diarrhoea }(n=2) \\
\text { Dehydration/anorexia/fatigue }(n=2)\end{array}$ \\
\hline
\end{tabular}

Table 4 Antitumour activity of i.v. and oral vorinostat

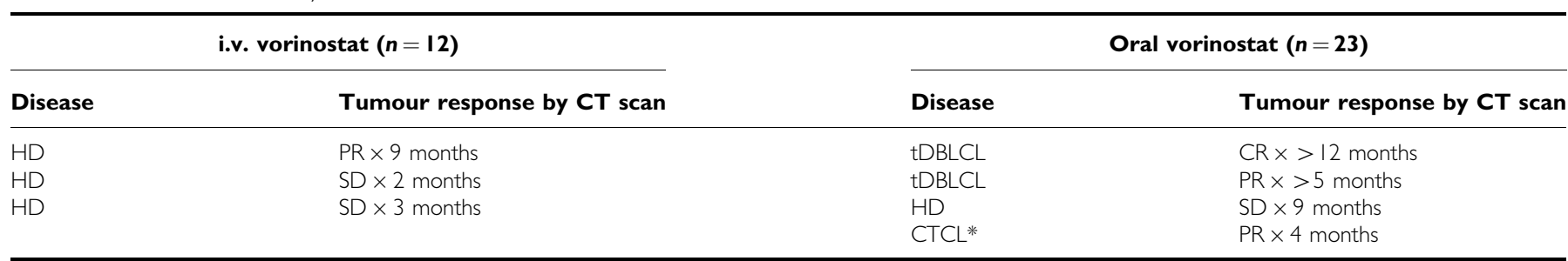

$\mathrm{CR}=$ complete remission; $\mathrm{CT}=$ computed tomography; $\mathrm{CTCL}=$ cutaneous T-cell lymphoma; $\mathrm{DLBCL}=$ diffuse large $\mathrm{B}$-cell lymphoma; HD = Hodgkin's disease; i.v. = intravenous; $\mathrm{PR}=$ partial response; $\mathrm{SAHA}=$ suberoylanilide hydroxamic acid; $\mathrm{SD}=$ stable disease. $*$ Response based on skin examination.

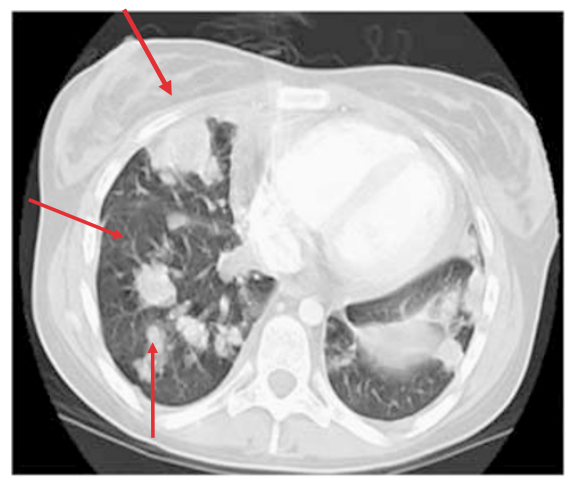

Baseline

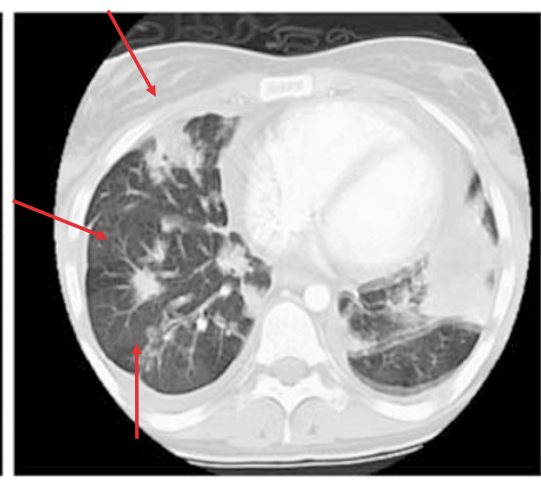

2-Month Follow-up

Figure 4 CT scan of thoracic cavity of a patient with refractory HD before and after i.v. vorinostat $600 \mathrm{mg} \mathrm{m}^{-2}$ for 5 days every 3 weeks.

Oral vorinostat also resulted in marked antitumour activity in a total of four patients, one with HD, two with transformed DBLCL, and one with cutaneous T-cell lymphoma (CTCL) (Table 4). The patient with HD showed a $31 \%$ tumour response lasting for 9 months, as assessed by CT scan. In the two patients with transformed DBLCL, both of whom were heavily pretreated before receiving vorinostat therapy, one experienced a CR lasting for 12 months and one experienced a PR lasting over 5 months. Figure 5 shows the resolution of a gastrohepatic mass over time in one of the patients with transformed DBLCL who received oral vorinostat. A substantial reduction in tumour size was apparent after one cycle of oral vorinostat therapy, and by two cycles, the mass had completely disappeared. This patient also demonstrated a negative PET scan performed after 5 months of therapy. The patient with CTCL exhibited a PR of his skin tumour over 4 months.

\section{CONCLUSIONS}

The phase I clinical trial experience in patients with haematological malignances showed that both i.v. and oral formulations of vorinostat are well tolerated. The DLTs of i.v. vorinostat were primarily haematologic toxicities, which were rapidly reversible within 4-5 days of therapy cessation. For oral vorinostat, the DLTs were primarily non-haematologic toxicities (dehydration, anorexia, diarrhoea, fatigue), which were also rapidly reversible with a median duration of 3 days. The differences in the types of DLTs observed between oral and i.v. vorinostat may result from dissimilarities in the pharmacokinetics of the two formulations. Intravenous vorinostat administration may give rise to higher $C_{\max }$ values and lower AUC values than oral vorinostat, and this may have important ramifications in terms of the spectrum of toxicities observed.

Evidence of inhibition of HDAC activity in both PBMNC and tumour tissue biopsies was apparent for up to $10 \mathrm{~h}$ following oral administration of vorinostat. Inhibition of HDAC activity was associated with objective tumour regression and symptomatic improvement (with little clinical toxicity) in patients with a variety of haematological malignances, including HD and select subtypes of NHL, including T-cell lymphomas. Further studies are needed to define relevant biologic end points that can be used as a guide to optimise the dosing schedule for vorinostat, particularly with respect to dose, timing of administration, and duration of therapy. At the molecular level, further research is needed to fully delineate the 


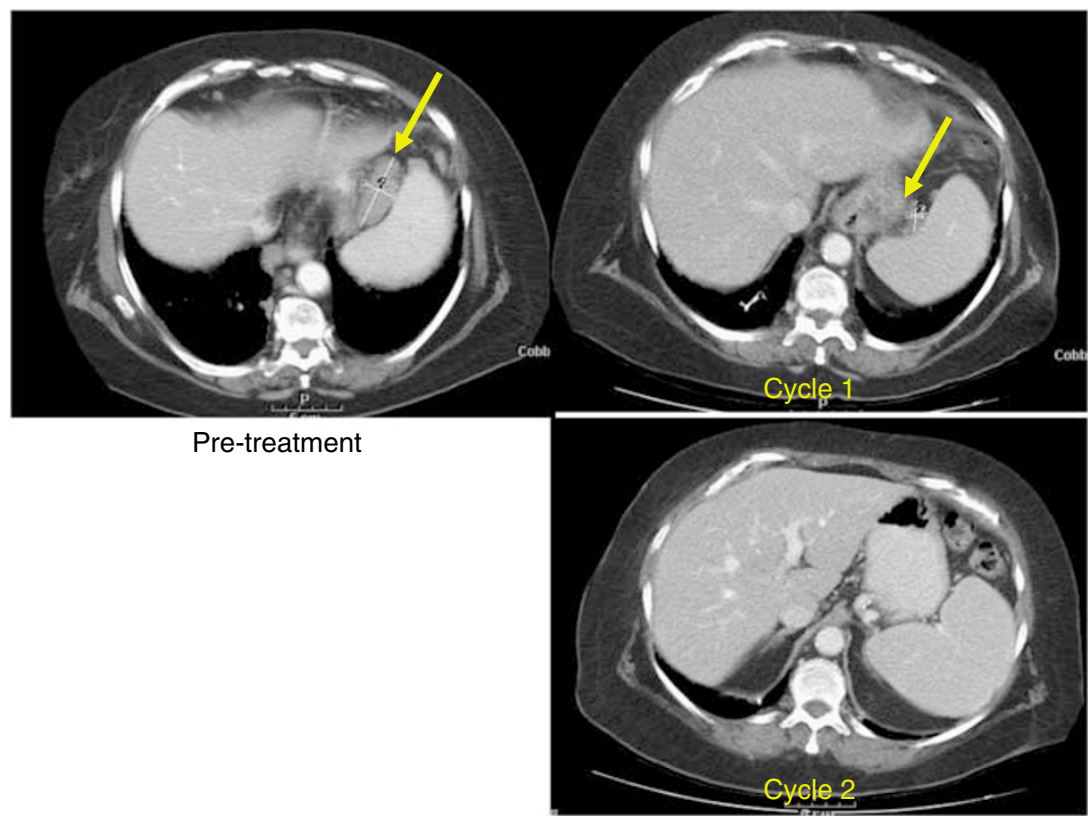

Figure 5 Resolution of a gastrohepatic mass in a patient with transformed DLBCL following oral vorinostat therapy. CT scans were taken before treatment and after one and two cycles of oral vorinostat.

mechanism(s) of antitumour effect of vorinostat in various types of malignances and to understand why normal cells are apparently more resistant to apoptosis than transformed cells. Several phase II studies are currently ongoing in patients with haematological malignances and solid tumours. The preliminary results of vorinostat's activity in T-cell lymphoma will be discussed by Dr Duvic.

\section{REFERENCES}

Bereshchenko OR, Gu W, Dalla-Favera R (2002) Acetylation inactivates the transcriptional repressor BCL6. Nat Genet 32: 606-613

Dokmanovic M, Marks PA (2005) Prospects: histone deacetylase inhibitors. J Cell Biochem 96: 293-304

Huang L, Pardee AB (2000) Suberoylanilide hydroxamic acid as a potential therapeutic agent for human breast cancer treatment. Mol Med 6: 849866

Kelly WK, O'Connor OA, Krug LM, Chiao JH, Heaney M, Curley T, Macgregore-Cortelli B, Tong W, Secrist JP, Schwartz L, Richardson S, Chu E, Olgac S, Marks PA, Scher H, Richon VM (2005) Phase I study of an oral histone deacetylase inhibitor, suberoylanilide hydroxamic acid, in patients with advanced cancer. J Clin Oncol 23: 3923-3931

Kelly WK, Richon VM, O'Connor O, Curley T, MacGregor-Curtelli B, Tong W, Klang M, Schwartz L, Richardson S, Rosa E, Drobnjak M, Cordon-Cordo C, Chiao JH, Rifkind R, Marks PA, Scher H (2003) Phase I clinical trial of histone deacetylase inhibitor: suberoylanilide hydroxamic acid administered intravenously. Clin Cancer Res 9: $3578-3588$

Kramer MH, Hermans J, Wijburg E, Philippo K, Geelen E, van Krieken JH, de Jong D, Maartense E, Schuuring E, Kluin PM (1998) Clinical relevance of BCL2, BCL6, and MYC rearrangements in diffuse large B-cell lymphoma. Blood 92: 3152-3162

Lo CF, Ye BH, Lista F, Corradini P, Offit K, Knowles DM, Chaganti RS, Dalla-Favera R (1994) Rearrangements of the BCL6 gene in diffuse large cell non-Hodgkin's lymphoma. Blood 83: 1757 - 1759

Marks P, Rifkind RA, Richon VM, Breslow R, Miller T, Kelly WK (2001) Histone deacetylases and cancer: causes and therapies. Nat Rev Cancer 1: $194-202$

\section{ACKNOWLEDGEMENTS}

$\mathrm{OAO}$ is the recipient of the Leukemia and Lymphoma Society Scholar in Research Award. Writing assistance for this paper was provided by Jan S. Redfern, $\mathrm{PhD}$, and funding was provided by Merck \& Co. Inc., Whitehouse Station, NJ 08889.

Marks PA, Dokmanovic M (2005) Histone deacetylase inhibitors: discovery and development as anticancer agents. Expert Opin Investig Drugs 14: $1497-1511$

Marks PA, Richon VM, Breslow R, Rifkind RA (1999) Hybrid polar inducers of transformed cell differentiation/apoptosis. From the cell to the clinic. CR Acad Sci III 322: $161-165$

Marks PA, Richon VM, Miller T, Kelly WK (2004) Histone deacetylase inhibitors. Adv Cancer Res 91: 137-168

Marks PA, Richon VM, Rifkind RA (2000) Histone deacetylase inhibitors: inducers of differentiation or apoptosis of transformed cells. J Natl Cancer Inst 92: 1210-1216

Munster PN, Troso-Sandoval T, Rosen N, Rifkind R, Marks PA, Richon VM (2001) The histone deacetylase inhibitor suberoylanilide hydroxamic acid induces differentiation of human breast cancer cells. Cancer Res 61: $8492-8497$

O'Connor OA, Heaney ML, Schwartz L, Richardson S, Willim R, MacGregor-Cortelli B, Curly T, Moskowitz C, Portlock C, Horwitz S, Zelenetz AD, Frankel S, Richon V, Marks P, Kelly WK (2006) Clinical experience with intravenous and oral formulations of the novel histone deacetylase inhibitor suberoylanilide hydroxamic acid in patients with advanced hematologic malignancies. J Clin Oncol 24: $166-173$

Pasqualucci L, Bereschenko O, Niu H, Klein U, Basso K, Guglielmino R, Cattoretti G, Dalla-Favera R (2003) Molecular pathogenesis of nonHodgkin's lymphoma: the role of Bcl-6. Leuk Lymphoma 44(Suppl 3): S5 - S12

Richon VM, Sandhoff TW, Rifkind RA, Marks PA (2000) Histone deacetylase inhibitor selectively induces p21WAF1 expression and 
gene-associated histone acetylation. Proc Natl Acad Sci USA 97: 1001410019

Richon VM, Webb Y, Merger R, Sheppard T, Jursic B, Ngo L, Civoli F, Breslow R, Rifkind RA, Marks PA (1996) Second generation hybrid polar compounds are potent inducers of transformed cell differentiation. Proc Natl Acad Sci USA 93: 5705-5708
Ye BH, Lista F, Lo CF, Knowles DM, Offit K, Chaganti RS, Dalla-Favera R (1993a) Alterations of a zinc finger-encoding gene, BCL-6, in diffuse large-cell lymphoma. Science 262: $747-750$

Ye BH, Rao PH, Chaganti RS, Dalla-Favera R (1993b) Cloning of bcl-6, the locus involved in chromosome translocations affecting band 3q27 in B-cell lymphoma. Cancer Res 53: 2732-2735 